\title{
Modellbasierte Selektion hyperspektraler EnMAP Kanäle zur optimalen Invertierung von Strahlungstransfermodellen für landwirtschaftliche Kulturen
}

\author{
Katja Berger $^{1} \cdot$ Clement Atzberger $^{2} \cdot$ Martin Danner $^{1} \cdot$ Matthias Wocher $^{1} \cdot$ Wolfram Mauser $^{1} \cdot$ Tobias Hank $^{1}$
}

Received: 10 December 2018 / Accepted: 22 January 2019 / Published online: 8 February 2019

(c) The Author(s) 2019

\begin{abstract}
Model-based Selection of hyperspectral EnMAP Channels for optimal Inversion of Radiation Transfer Models in Agriculture. Satellite-based hyperspectral Earth observation data combined with physically based radiative transfer models have the strong potential to support sustainable agriculture by providing accurate spatial and temporal information of important vegetation biophysical and biochemical variables such as leaf chlorophyll content. To meet this goal, possible error sources in the modelling should be minimized. Thus, the capability of a model to reproduce the measured spectral signals has to be tested before applying any retrieval algorithm. For an exemplary demonstration, the PROSAIL model was employed to emulate the setup of the future EnMAP hyperspectral sensor in the visible and near-infrared (VNIR) spectral region with a $6.5 \mathrm{~nm}$ spectral sampling distance. Model uncertainties were determined to subsequently exclude those wavelengths with highest mean absolute error (MAE) between model simulation and spectral measurement. For this purpose data from two campaigns were exploited (1) from Nebraska-Lincoln (maize and soybean) and (2) from Munich-North-Isar (maize and winter wheat). A significant increase of accuracy for leaf chlorophyll content (LCC, $\mu \mathrm{g} \mathrm{cm}^{-2}$ ) estimations could be obtained, with relative RMSE decreasing from $26 \%$ (full VNIR range) to 15\% (optimized VNIR) for maize and from $77 \%$ to $29 \%$ for soybean, respectively. We therefore recommend applying a specific model-error threshold (MAE 0.01) to stabilize the retrieval of crop biochemical variables.
\end{abstract}

Keywords EnMAP $\cdot$ PROSAIL $\cdot$ LAI $\cdot$ Leaf chlorophyll content $\cdot$ Radiative transfer model $\cdot$ Agriculture

\section{Zusammenfassung}

Zukünftige hyperspektrale Erdbeobachtungsmissionen, kombiniert mit physikalisch basierten Strahlungstransfermodellen, haben ein großes Potential eine nachhaltige Landwirtschaft zu unterstützen. Anhand dieser neuen Aufnahmen können noch genauere räumliche und zeitliche Informationen über wichtige biophysikalische und biochemische Variablen der Vegetation, wie beispielsweise den Blattchlorophyllgehalt, zur Verfügung gestellt werden. Allerdings müssen dafür mögliche Fehlerquellen in der Modellierung minimiert werden. Bevor ein Schätz- bzw. Inversionsalgorithmus angewendet wird, sollte zunächst die Fähigkeit des Modells zur Simulation der gemessenen spektralen Signatur getestet werden. In der aktuellen Studie wurde das weit verbreitete PROSAIL-Modell verwendet, um den zukünftigen EnMAP Hyperspektralsensor im sichtbaren und nahen Infraroten Spektralbereich (VNIR) mit einer spektralen Auflösung von 6,5 nm zu simulieren. Die Abweichungen zwischen Modell und gemessenen Daten wurden beispielhaft an zwei Kampagnen und drei verschiedenen Feldfrüchten (Mais, Winterweizen und Sojabohnen) demonstriert. Diejenigen Wellenlängen mit dem höchsten mittleren absoluten Fehler (MAE) zwischen der Modellsimulation und der Spektralmessung wurden vollautomatisiert mit einem sequentiellen Look-up Table (LUT) basierten Algorithmus (Feature-Selektions-Algorithmus) ausgeschlossen. Für die Bestimmung des Blattchlorophyllgehalts (LCC, $\mu \mathrm{g} \mathrm{cm}^{-2}$ ) der Nebraska-Kampagne konnten signifikante Verbesserungen der Schätzung erreicht werden: im Fall von Mais sank der relative RMSE (rRMSE) von 26\% (gesamter VNIR-Bereich) auf 15\% (optimierter VNIR-Bereich), und für Sojabohnen von rRMSE $=77 \%$ auf rRMSE $=29 \%$. Auf Grund dieser positiven Ergebnisse empfehlen wir die Anwendung eines spezifischen ModellfehlerSchwellenwerts (MAE 0.01) für den spektralen VNIR Bereich. In diesem Rahmen sollten die spektralen Messungen und Modellsimulationen mindestens übereinstimmen, um eine vertrauenswürdige Schätzung biochemischer Vegetationsvariablen aus zukünftigen Hyperspektralmissionen zu garantieren.

Extended author information available on the last page of the article 


\section{Einleitung}

Weltweit besteht ein zunehmender Bedarf landwirtschaftliche Bewirtschaftungssysteme $\mathrm{zu}$ optimieren, einerseits um Erträge zu steigern und andererseits, um mögliche Umweltauswirkungen zu minimieren (Godfray et al. 2010). Erdbeobachtung anhand von Satelliten wird zunehmend für die sogenannte Präzisionslandwirtschaft (Precision Farming) eingesetzt. Dabei werden flächendeckend und kontinuierlich biophysikalische und biochemische Informationen zu Oberflächen- bzw. Vegetationseigenschaften, z.B. Blattflächenindex (LAI, in $\mathrm{m}^{2} \mathrm{~m}^{-2}$ ) oder Blattchlorophyllgehalt (LCC, in $\mu \mathrm{g} \mathrm{cm}{ }^{-2}$ ) bereitgestellt (D'Urso et al. 2010). Derzeit sind mehrere hyperspektrale Satellitenmissionen in Planung, u.a. das U.S. amerikanische Hyperspectral Infrared Imager (HyspIRI) der NASA (Lee et al. 2015), das hyperspektrale Erdbeobachtungsinstrument DESIS (Eckardt et al. 2015) des deutschen Luft- und Raumfahrtzentrums (DLR), der italienische Hyperspectral Precursor of the Application Mission (PRISMA, PRecursore IperSpettrale della Missione Applicativa) der Agenzia Spaziale Italiana (ASI) (Labate et al. 2009) sowie das deutsche "Environmental Mapping and Analysis Program" (EnMAP) (Guanter et al. 2015). Durch die verbesserte zeitliche, räumliche und vor allem spektrale Auflösung kann in Zukunft der Zustand sowie die Dynamik der Vegetation großflächig und mit einer höheren Genauigkeit als mit den derzeit verfügbaren multispektralen Systemen aufgenommen werden (Hank et al. 2018).

Der Start der ersten deutschen hyperspektralen Satellitenmission EnMAP ist für 2020 geplant. Der Sensor bietet eine vollständige VNIR-SWIR-Spektralabdeckung mit circa 242 Spektralkanälen und $30 \mathrm{~m}$ räumlicher Auflösung mit einem hohen Signal-Rausch-Verhältnis und einer Wiederholrate von 4 Tagen (Schwenkung $\pm 30^{\circ}$ ), bzw. bis zu 27 Tagen (Schwenkung $\pm 5^{\circ}$ ). Die zunehmende Verfügbarkeit von Hyperspektraldaten fördert die Entwicklung von generischen und übertragbaren Retrieval-Techniken (RiveraCaicedo et al. 2017; Verrelst et al. 2018). Angesichts des hohen spektralen Informationsgehaltes der neuen Sensoren sind physikalisch basierte Strahlungstransfermodelle ideal für die Analyse und Auswertung dieser Daten (Vohland and Jarmer 2008; Vohland et al. 2010). Dies kann zum Beispiel durch Verwendung des bekannten und weit verbreiteten, bidirektionalen Reflexionsmodells 4SAIL (Verhoef et al. 2007) und des optischen Blattmodells PROSPECT-D (Féret et al. 2017) realisiert werden. Die kombinierte Modellversion, allgemein als ,PROSAIL' bezeichnet (Jacquemoud et al. 2009), stellt einen idealen Kompromiss zwischen Komplexität und Recheneffizienz dar, wodurch sich das Modell insbesondere für die Verarbeitung größerer
Datenmengen im landwirtschaftlichen Bereich eignet (Berger et al. 2018). Um die benötigten biophysikalischen oder biochemischen Variablen zu schätzen, muss eine geeignete Modell-Inversionsstrategie gewählt werden. Aktuelle Ansätze des maschinellen Lernens stellen hierbei eine der effektivsten und genauesten Möglichkeiten zur Schätzung der Variablen dar (Rivera-Caicedo et al. 2017; Verrelst et al. 2018). Das ist insbesondere dann der Fall, wenn sie auf großen synthetischen Modelldatensätzen trainiert werden (=hybride Methode), wie sie zum Beispiel durch PROSAIL erstellt werden. So genannte Look-up Tables (LUT) werden auch in der aktuellen Studie aufgegriffen. Sie stellen eine der häufigsten Inversionsmethoden dar (Kimes et al. 2000; Locherer et al. 2015; Weiss et al. 2000). Dabei wird eine definierte Anzahl (z.B. 100.000) von Parameterkombinationen gemeinsam mit der korrespondierenden spektralen Reflexion für einen definierten Wellenlängenbereich, bzw. eine bestimmte Sensorkonfiguration abgespeichert. Das Inversionsergebnis für ein gegebenes Pixel entspricht dann der Parameterkombination des LUT, welche die beste Übereinstimmung zwischen simulierter und gemessener spektraler Signatur erzielt.

Nicht alle Bestandsvariablen sind gleichermaßen genau schätzbar. Im Vergleich zur Schätzung von strukturellen Bestandsvariablen wie dem LAI stellt sich z.B. die Schätzung biochemischer Blattvariablen aus dem Bestandssignal oftmals als besonders schwierig heraus (Botha et al. 2010; Darvishzadeh et al. 2008). Die Eigenschaften der Hyperspektraldaten erlauben, die im Vegetationsspektrum subtil auftretenden Signale besser zu identifizieren und damit verbundene Blattvariablen (z.B. Pigmente) mit höherer Genauigkeit zu schätzen. Außerdem gibt es auf verschiedenen Ebenen Möglichkeiten, die Schätzgenauigkeit aus den Modellen zu unterstützen (Baret and Buis 2008; Dorigo et al. 2009), z.B.:

- Auswahl besonders sensitiver Spektralbereiche: Einschränkung der Analyse auf Spektralbereiche, in denen die gesuchte Variable das spektrale Signal dominiert (Verger et al. 2011)

- Vordefiniertes Sampling: Definition einer Verteilung der Variablen in der LUT, z.B. uniforme oder Gaußsche Normalverteilung in einem bestimmten Parameterbereich. Diese Informationen basieren meistens auf a priori Wissen durch Felddatenerhebung oder Berechnung anhand von Wachstumsmodellen (Baret and Buis 2008; Verger et al. 2011)

- Nachbarschaft: Hierbei wird die Tatsache genutzt, dass die Charakteristika nahe beieinander liegender Objekte ähnlicher sind als die weiter entfernt liegender Objekte (Atzberger and Richter 2012a, b)

- Zeitliche Einschränkungen: Nutzbarmachung der zeitlichen Autokorrelation von Parametern zur 
nachträglichen Optimierung der Kostenfunktion (Baret and Buis 2008; Koetz et al. 2005)

Die genannten Maßnahmen können die Kartierung der Vegetationsgrößen aber nur unterstützen, wenn die Voraussetzungen von fehlerfreien Messungen und Modellsimulationen gegeben sind. Ein Nachteil der physikalisch basierten oder hybriden Inversionsansätze - insbesondere im Rahmen der Auswertung von hyperspektralen Daten - ist ihre Empfindlichkeit gegenüber korrupten oder weniger gut modellierten (und gemessenen) Spektralkanälen (Atzberger et al. 2013; Meroni et al. 2004). Daher ist es erforderlich, zunächst die Fähigkeit des Modells zu testen, das spektrale Signal der Vegetation zu reproduzieren, bevor man es zur Schätzung der biophysikalischen bzw. biochemischen Variablen einsetzt. Bereits geringfügige Abweichungen zwischen modellierter und gemessener Reflexion können das Ergebnis (negativ) beeinflussen. In einer Studie von Atzberger et al. (2013) wurde dies beispielhaft für Grasflächen gezeigt: Anhand eines automatisierten Feature-Selektions-Algorithmus (FS-Algorithmus) wurden schlecht modellierte Wellenlängen sequentiell identifiziert und ausgeschlossen, was letztlich zu einer genaueren Schätzung der Variablen führte. In einer Studie, die verschiedene Feldfrüchte anhand eines multispektralen Datensatzes analysierte (Atzberger und Richter 2012a, b), wurden drei von sechs Feldfrüchten (einschließlich Mais) verworfen, da die Simulation mit PROSAIL im VorwärtsModus (parametrisiert durch Feldmessungen) nicht mit den beobachteten Signaturen übereinstimmte. Die zukünftige Verfügbarkeit von Hyperspektraldaten wird eine genauere Identifizierung von modellierten und gemessenen spektralen Unsicherheiten ermöglichen (Hank et al. 2018). Im Hinblick auf die komplexen und variierenden Bestandsstrukturen auf landwirtschaftlichen Flächen sollten solche FS-Algorithmen an verschiedenen Feldfrüchten, an diversen Standorten sowie anhand aktueller Sensoren während der gesamten Vegetationsperiode eingesetzt werden.

Das Ziel der vorliegenden Studie war einerseits, die Fähigkeit des PROSAIL-Modells zu untersuchen und hyperspektrale Messungen im LCC - sensitiven Spektralbereich $(400-700 \mathrm{~nm})$ für Weizen, Mais und Sojabohne zu reproduzieren. Im Weiteren sollte die Auswirkung weniger gut reproduzierter Kanäle, bzw. deren Nichtberücksichtigung bei der Modellierung, für die Kartierung des Blattchlorophyllgehalts analysiert werden. Insgesamt wurde das Ziel verfolgt, die Verwendbarkeit von PROSAIL-Simulationen für wichtige Kulturarten als Voraussetzung für die Anwendung des Modells innerhalb einer automatisierten Prozedur zur Kartierung biochemischer Variablen aus zukünftigen hyperspektralen Sensormissionen zu verbessern.

\section{Material}

Daten von zwei Feldkampagnen wurden im Rahmen dieser Studie analysiert: (1) Nebraska-Lincoln und (2) MünchenNord-Isar. Ausführliche Informationen zu Messprotokoll und Berechnungen der ersten Kampagne sind bei Ciganda et al. (2008) und Gitelson et al. (2003) zu finden. In Tab. 1 wird eine Übersicht der gemessenen Variablen der beiden Feldkampagnen gezeigt.

\subsection{Nebraska-Lincoln (UNL-ARDC) Kampagne}

Der Testdatensatz der vorliegenden Studie wurde in den Vegetationsperioden 2001 bis 2008 am Landwirtschaftlichen Forschungs- und Entwicklungszentrum der Universität Nebraska-Lincoln (Carbon Sequestration Program at the University of Nebraska-Lincoln Agricultural Research and Development Center, UNL-ARDC) erhoben. Die Untersuchungen wurden an drei landwirtschaftlichen Feldern circa $58 \mathrm{~km}$ nordöstlich von Lincoln, NE, USA, durchgeführt (Viña et al. 2011). Dabei handelte es sich (1) um ein dauerhaft bewässertes Maisfeld, (2) um eine bewässerte MaisSojabohnenrotation $\left(41^{\circ} 09^{\prime} 50^{\prime \prime} \mathrm{N}, 9^{\circ} 28^{\prime} 40^{\prime \prime} \mathrm{W}, 361 \mathrm{~m}\right)$ sowie (3) um eine Mais-Sojabohnenrotation unter Regenfeldbau $\left(41^{\circ} 10^{\prime} 50^{\prime \prime} \mathrm{N}, 96^{\circ} 26^{\prime} 20^{\prime \prime} \mathrm{W}, 362 \mathrm{~m}\right)$ von jeweils circa 65 ha Größe.

Unter Verwendung eines Zweifaseroptiksystems (Ocean Optics USB2000-Radiometer) wurden spektrale Daten von 400 - $900 \mathrm{~nm}$ mit einem spektralen Sampling von $0.3 \mathrm{~nm}$ über den Feldfrüchten aufgenommen (18 - 34 Kampagnen pro Jahr von Anfang Mai/Juni bis circa September des jeweiligen Jahres). Die spektralen Aufnahmen wurden sogenannten intensiven Mess-Zonen (IMZ) gleicher Größe zugeordnet. An jeder IMZ wurden strukturelle Vegetationsmessungen (LAI, $\mathrm{m}^{2} \mathrm{~m}^{-2}$ ), sowie biochemische Untersuchungen des Blattchlorophyllgehalts ( $\mathrm{LCC}, \mu \mathrm{g} \mathrm{cm}^{-2}$ ) durchgeführt (Ciganda et al. 2008; Viña et al. 2011). Die Blattchlorophyllgehalte wurden anhand einer Beziehung zwischen Blattreflexion und Pigmentgehalt berechnet, die

Tab. 1 Übersicht der Messungen des Blattchlorophyllgehalts von Mais und Sojabohnen im Nebraska-Lincoln-Untersuchungsgebiet sowie von Mais und Winterweizen im München-Nord-Isar (MNI) Testgebiet: Gesamtzahl (N), Range (Min - Max), Mittelwert, Standardabweichung (SD) und Variationskoeffizient (CV)

\begin{tabular}{lrrrrr}
\hline $\begin{array}{l}\text { Blattchlorophyll- } \\
\text { gehalt }\left(\mu \mathrm{g} \mathrm{cm}^{-2}\right)\end{array}$ & N & Min - Max & Mean & SD & CV \\
\hline Mais Nebraska & 169 & $19,5-83,0$ & 56,1 & 9,9 & 0,18 \\
Soja Nebraska & 68 & $11,8-58,2$ & 30,3 & 8,9 & 0,30 \\
Mais MNI & 26 & $27,3-61,6$ & 46,2 & 11,4 & 0,15 \\
Weizen MNI & 40 & $11,6-59,5$ & 45,0 & 11,7 & 0,26 \\
\hline
\end{tabular}


auf dem Red Edge Chlorophyll Index basiert. Dafür wurde die individuelle Blattreflexion im Spektralbereich von $400 \mathrm{~nm}$ bis $900 \mathrm{~nm}$ mit einem Blattclip am Ocean Optics USB2000Radiometer gemessen.

Das geplante spektrale Sampling des zukünftigen EnMAPSensors wird im sichtbaren und nahen Infrarot $6,5 \mathrm{~nm}$ betragen und somit einen Kompromiss zwischen mehreren zukünftigen Hyperspektralsensoren darstellen (von DESIS mit 2,55 nm bis PRISMA mit $12 \mathrm{~nm}$ ). Aus diesem Grund wurden EnMAP bandspezifische Responsefunktionen auf die Feldspektrometer-Daten im VNIR-Bereich angewendet $\left(\mathrm{N}_{\text {samples }}=73\right.$ im Spektralbereich von 423 bis $863 \mathrm{~nm}$ ). Aufgrund des stärker verrauschten Signals der Spektrometer-Daten $>870 \mathrm{~nm}$ wurden diese Wellenlängen von der Berechnung ausgeschlossen. Die verfügbare spektrale Information reicht aber für die Betrachtung des Blattchlorophyllgehalts vollkommen aus. Insgesamt standen 169 Messungen von LCC in $\left[\mu \mathrm{g} \mathrm{cm}^{-2}\right]$ für Mais und 68 Proben für Sojabohnen zur Verfügung.

\subsection{München-Nord-Isar (MNI) Kampagne}

Für Demonstrationszwecke wurde außerdem ein umfassender Datensatz aus dem Untersuchungsgebiet München-Nord-Isar (MNI) in Bayern, Süddeutschland, herangezogen. In den Jahren 2014, 2015, 2017 und 2018 wurden die Messstandorte während der Wachstumsperiode von März bis September regelmäßig aufgesucht. Felder des Münchner kommunalen Farmlands östlich der Isar wurden für die Analysen ausgewählt. Die exakten Standorte der Probennahmen variierten von Jahr zu Jahr aufgrund der wechselnden Fruchtfolge. Alle Felder befanden sich jedoch in einem Umkreis von $5 \mathrm{~km}$ Durchmesser, zentriert um $48^{\circ} 16^{\prime} 04^{\prime \prime} \mathrm{N}, 11^{\circ} 42^{\prime} 45^{\prime \prime} \mathrm{E}$ (Wocher et al. 2018). Jeder Standort war auf eine Fläche von $30 \mathrm{~m}$ x $30 \mathrm{~m}$ beschränkt und wurde in neun IMZs unterteilt. Bestandsspektren wurden im Nadir-View anhand eines ASD FieldSpec 3 Jr. (Boulder, CO, USA) aufgenommen. Messungen erfolgten nur unter wolkenfreien Bedingungen. Spektrale Messungen wurden im Bereich von $350-2500 \mathrm{~nm}$ aufgenommen. Neben den Top-of-Canopy (TOC) Spektren wurde für jeden Termin auch ein repräsentatives Bodenspektrum aufgenommen, das dem Modell als Hintergrundinformation dienen sollte. Ein Konica-Minolta SPAD-502 Gerät diente zur Messung interner SPAD-Einheiten, die über eine Kalibrierungskurve in Blattchlorophyll $\mathrm{a}+\mathrm{b}$ (LCC) umgewandelt wurden (SPAD $=$ single-photon avalanche diode). Von LCC wurde auch der Karotinoidgehalt $\left(\mathrm{C}_{\mathrm{cx}}\right)$ mittels eines linearen Regressionsmodells abgeleitet, da LCC und $\mathrm{C}_{\mathrm{cx}}$ im Fall von gesunder grüner Vegetation stets eine stabile Beziehung aufweisen (Baret et al. 1988). Für die vorliegende Studie standen 26 LCC Messungen für Mais sowie und 40 für Winterweizen (LCC in $\mu \mathrm{g} \mathrm{cm}^{-2}$ ) zur Verfügung (Tab. 1).

\section{Methodik}

\subsection{Feature Selektion}

Von jeder Feldfrucht wurden 20 Beispiele (d.h. 20 IMZs) ausgewählt, um die Übereinstimmung zwischen simulierten und gemessenen Spektren zu beurteilen. Die 20 IMZs wurden jeweils über die gesamte Vegetationsperiode extrahiert, um den Einfluss der Phänologie zu minimieren. Im nächsten Schritt wurde für jede IMZ eine individuelle LUT (LUT ${ }_{\text {ind }}$ ) mit dem PROSAIL-Modell erstellt. Hierfür wurde der LAI auf die jeweils gemessenen Werte festgelegt, da diese Strukturvariable den größten Einfluss auf das Gesamtspektrum hat, neben dem Blattwinkel, der aber nicht gemessen wurde. Alle anderen Modellparameter konnten innerhalb einer gleichmäßigen Verteilung frei variieren. Dazu gehört der N-Parameter $(1.2-2.6)$, LCC mit $0-85 \mu \mathrm{g} \mathrm{cm}{ }^{-2}$, Blatt-Trockenmassegehalt $\left(\mathrm{C}_{\mathrm{m}}\right)$ mit $0.001-0.02 \mathrm{~g} \mathrm{~cm}^{-2}$, BlattWassergehalt (EWT) mit $0.001-0.05 \mathrm{~cm}$, Blatt-KarotinoidGehalt $\left(\mathrm{C}_{\mathrm{cx}}\right)$ mit $0-20 \mu \mathrm{g} \mathrm{cm}{ }^{-2}$, Blatt-Anthocyan-Gehalt $\left(\mathrm{C}_{\text {anth }}\right)$ mit $0-2 \mu \mathrm{g} \mathrm{cm}{ }^{-2}$, mittlerer Blattwinkel (ALIA) mit $30-70^{\circ}$, Hot-Spot Parameter mit $0.01-0.03 \mathrm{~m} \mathrm{~m}^{-1}$. Diese Werte der Parameter wurden durch die Erfahrung der Autoren mit dem PROSAIL Modell sowie entsprechend der Literatur festgelegt (Atzberger et al. 2013; Berger et al. 2018; España et al. 1999; Féret et al. 2017; Richter et al. 2009; Verrelst et al. 2016).

Hintergrundspektren (Bodenreflexion) sowie die aktuelle Mess- und Beleuchtungsgeometrie wurden entsprechend den tatsächlichen Bedingungen festgelegt. Für jede LUT $_{\text {ind }}$ wurden Spektralsignale für 10.000 zufällig ausgewählte Parameterkombinationen simuliert. Simulierte PROSAILReflexionen wurden in $\mathrm{N}_{\text {Bands }}=73$ VNIR-Bänder umgewandelt, wobei die tatsächlichen bandspezifischen Responsefunktionen des zukünftigen EnMAP Sensors verwendet wurden (mittlere Wellenlängen von $423 \mathrm{~nm}$ bis $863 \mathrm{~nm}$ ). Für jede einzelne IMZ wurde nun anhand der Wurzel der mittleren Fehlerquadratsumme (RMSE) das am besten passende LUT-Spektrum ausgewählt. Im nächsten Schritt wurde die Differenz zwischen diesem LUT Spektrum und der jeweiligen Messung berechnet. Am Schluss wurde der Spektralkanal mit den höchsten Abweichungen aller 20 IMZs einer Feldfrucht identifiziert, d.h. mittlerer absoluter Fehler und MAE. Dieser Kanal wurde gelöscht und die Prozedur mit N-1 Spektralkanälen wiederholt. Die daraus resultierenden spezifischen Bandkombinationen des Nebraska Datensatzes wurden dann für die Schätzung des gesamten Blattchlorophyll Datensatzes (LCC in $\mu \mathrm{g} \mathrm{cm}^{-2}$ ) verwendet, d.h. für Mais $N=169$, und für Sojabohnen $N=68$. Für die vorliegende Publikation wurden die Daten der MNI Kampagne ausgeschlossen, da sie bereits in einer anderen Studie ausgewertet und analysiert wurden. 


\subsection{LUT Invertierung}

Für die LUT-basierte Inversion des PROSAIL-Modells folgten wir Standardansätzen (Darvishzadeh et al. 2008; Richter et al. 2009; Weiss et al. 2000), leicht modifiziert durch das bereits erwähnte Sampling Constraint: LCC wurde entsprechend einer Gauß'schen Normalverteilung charakterisiert, und somit den Werten angenähert, die bei der Feldkampagne erhoben wurden (d.h. für Mais: Mittelwert $(\mu)=50 \mu \mathrm{g} \mathrm{cm}^{-2}$, Standardabweichung (SD) $=20 \mu \mathrm{g} \mathrm{cm}^{-2}$; Sojabohnen: $\mu=30 \mu \mathrm{g} \mathrm{cm}^{-2}$ und $\mathrm{SD}=20 \mu \mathrm{g} \mathrm{cm}^{-2}$ ). Solche Informationen sind zwar nicht in jedem Fall verfügbar, können aber auch aus der Expertise abgeleitet werden. Alle anderen Parameter wurden wie in Kapitel 3.1 eingesetzt. Die Gesamtgröße der LUT wurde auf 20.000 Kombinationen von Modelleingangsparametern erweitert und die entsprechende bidirektionale Reflexion anhand des PROSAIL-Modells unter Verwendung der spektralen Responsefunktionen von EnMAP berechnet. Um die Lösung für das inverse Problem eines gemessenen Spektrums zu finden, wurde wiederum der mittlere RMSE zwischen dem gemessenen und jedem einzelnen modellierten Spektrum berechnet. Im Gegensatz zum LUT $_{\text {ind }}$ wurde aber nicht das Spektrum mit dem kleinsten RMSE als Ergebnis angesehen. Um eventuelle Modellunsicherheiten zu berücksichtigen, wurden der Mittelwert der 50 Spektren bzw. der LCC Werte mit dem kleinsten RMSE als endgültige Lösung ausgewählt.

\section{Ergebnisse \& Diskussion}

\subsection{Modelltest und Feature Selektion}

Abbildung 1 zeigt die spektralen Abweichungen (d.h. MAE) von 20 IMZs zwischen gemessenen und simulierten Bestandsspektren für Mais (o.l.) und Sojabohne (o.r.) der Nebraska Kampagne sowie für Mais (u.l.) und Winterweizen (u.r.) der MNI Kampagne. Kulturübergreifend sind Ähnlichkeiten erkennbar (d.h. 420 - $500 \mathrm{~nm}$ negative, ab $500 \mathrm{~nm}$ positive, und ab $650 \mathrm{~nm}$ meistens wieder negative Abweichungen). Allerdings ist die Ähnlichkeit der Fehler an einem Standort trotz verschiedener Bestandsstruktur größer als beim Betrachten der gleichen Feldfrucht (Mais). Dies zeigt, wie stark solche Messungen von den Standortgegebenheiten und den verwendeten Messinstrumenten abhängen. Im Allgemeinen sind bei Mais die größten Abweichungen und beim Winterweizen die geringsten zu finden. Beispielsweise wurde der spektrale Bereich von $580 \mathrm{~nm}$ bis $640 \mathrm{~nm}$ deutlich besser für Sojabohnen und Weizen als für Maispflanzen simuliert.

Die Ähnlichkeit im Muster der drei Feldfrüchte deutet darauf hin, dass das PROSAIL Modell generell Schwierigkeiten hat, bestimmte Spektralbereiche
- unabhängig von der Feldfrucht - zu simulieren. Dies wurde auch durch andere Studien bestätigt, zum Beispiel für Grünland (Darvishzadeh et al. 2008). In der Abb. 4 dieser Studie kann ein ähnliches Muster für den Bereich von 500 - $600 \mathrm{~nm}$ identifiziert werden. Die Unterschiede zwischen Feldfrüchten können dagegen auf kulturspezifisches Reflexionsverhalten zurückgeführt werden, das sich auch während der Vegetationsperiode ändert (Anteil Boden, Blüten, Früchte, Seneszenz, etc.). Die recht einfache Parametrisierung von PROSAIL reicht möglicherweise nicht aus, um diese Änderungen der Bestandsstruktur adäquat zu beschreiben (Doktor et al. 2014). Die Studie Atzberger and Richter (2012a) verwarf drei von sechs Feldfrüchten, darunter Mais, da die Vorwärtsmodellierung nicht den beobachteten Signaturen eines flugzeuggetragenen Sensors entsprach. Die Autoren erklärten dies mit den starken Reiheneffekten, welche das 1D-Modell PROSAIL nicht berücksichtigt könne. Zum Beispiel beeinflusst ein frühes Wachstumsstadium mit relativ hohem Bodenanteil das vom Sensor aufgenommene Signal zu stark, um genaue Schätzungen der Variablen zu ermöglichen.

Die Ergebnisse der Feature Selektion für den Nebraska Datensatz sind in Abb. 2 zusammengefasst. Anfänglich nimmt der mittlere absolute Fehler zwischen gemessener und simulierter Reflexion parallel zur abnehmenden Anzahl von Bändern stark ab, siehe Abb. 2 (links). Dieser Effekt wird bei Mais auf einem höheren Niveau (max. MAE $=0.0108$ ) als bei Sojabohnen $(\max . \mathrm{MAE}=0.0103)$ beobachtet. Interessanterweise konvergieren die Kurven beider Kulturen an einem bestimmten Punkt (MAE $=0.009)$ und nehmen dann linear $a b$, bis der MAE $=0$ ist. Die Verwendung genau dieser spektralen Bandkombinationen führt zu der Schätzgenauigkeit aus Abb. 2 (rechts). Das Entfernen der Spektralkanäle mit der schlechtesten Übereinstimmung führte zuerst auch zu einer sehr starken Verbesserung der Schätzgenauigkeit. Sobald der optimale RMSE erreicht ist, bleibt er stabil im Fall von Sojabohnen, bzw. nimmt wieder zu (Mais). Es scheint also, dass die Bandkombination des optimalen RMSE eine Abhängigkeit von der Feldfrucht aufweist. Daher kann eine Verallgemeinerung des Modellfehler Schwellenwertes (MAE) zwischen gemessen und simulierten Spektren für alle Feldfrüchte nur durch einen Kompromisswert erreicht werden, bei dem der spektrale Fehler angemessen und die Schätzgenauigkeit trotzdem zufriedenstellend ist. In Abb. 2 wäre dies circa beim spektralen Sampling von $\mathrm{N}_{\text {bands }} \sim 62 \mathrm{der}$ Fall, wobei ein mittlerer absoluter Fehler von MAE $\sim 0.01$ im VNIR-Bereich erreicht wird. In der Studie von Atzberger et al. (2013) wurde ein Schwellwert von MAE $\leq 0.02$ vorgeschlagen. Dieser beruhte jedoch auf einem größeren Gesamtspektrum, das bis zum mittleren Infrarot (SWIR) reichte. In der aktuellen Chlorophyll-bezogenen Studie widmen wir uns aber nur dem sichtbaren und nahen Infrarot (VNIR). Eine Untersuchung des gesamten Spektrums von $400-2500 \mathrm{~nm}$ (wie auch vom EnMAP Sensor abgedeckt) 
Abb. 1 Mittlerer absoluter Fehler zwischen gemessenem und simulierter Reflexion, Mittelwerte für jeweils 20 IMZs von Mais (o.l.) und Sojabohnen (o.r.) der Nebraska Kampagne, von Mais (u.1.) und Winterweizen (u.r.) der MNI Kampagne
Abb. 2 PROSAIL-Verhalten beim Anwenden einer sequentiellen Bandentfernung, d. h. eines sequentiellen Entfernens der am wenigsten genau simulierten Kanäle während des $\mathrm{LUT}_{\text {ind }}$-Laufs. Links: mittlerer absoluter Fehler zwischen gemessenen und simulierten Spektren. Rechts: Verwendung der entsprechenden KanalKombination für die Schätzung von LCC $\left[\mu \mathrm{g} \mathrm{cm}^{-2}\right]$. Für die Analysen wurde der gesamte Nebraska Datensatz mit den $\mathrm{N}=169$ Mais (grün) und $\mathrm{N}=68$ Sojabohnen (blau) verwendet
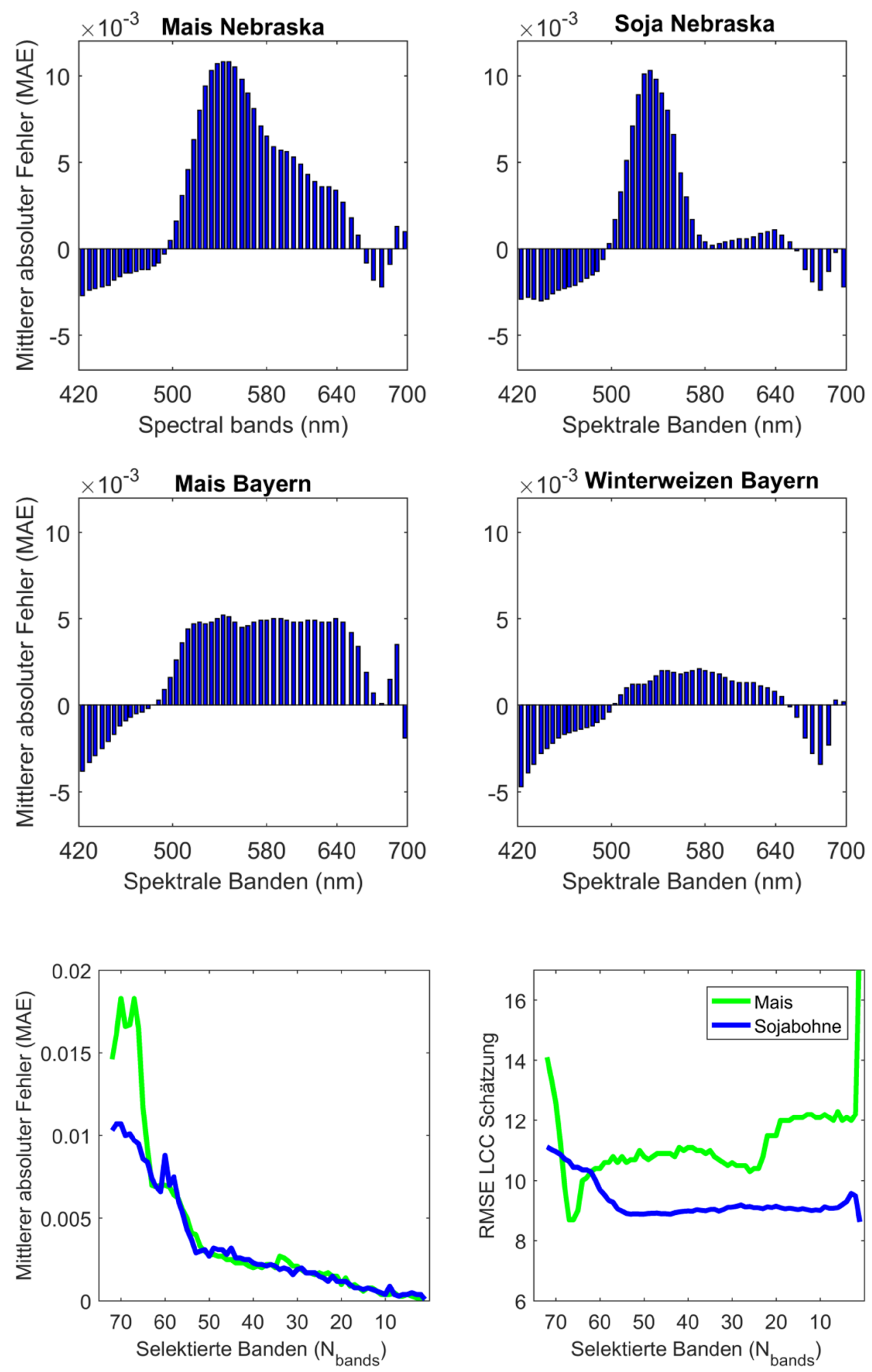

ist $\mathrm{zu}$ empfehlen, wenn weitere wichtige biochemische und biophysikalische Variablen untersucht werden, wie z.B. LAI, ALIA, EWT oder Proteingehalt.

\subsection{Schätzung des Blattchlorophyllgehalts}

Die Feature Selektion zeigte bereits in Abb. 2 (rechts) einen beachtlichen Einfluss auf die Schätzung des Blattchlorophyllgehalts. In Abb. 3 wird dies noch deutlicher: 
Während die Verwendung aller verfügbaren VNIR-Banden $\left(\mathrm{N}_{\text {bands }}=73\right) \mathrm{zu}$ einer Überschätzung und Sättigung im Fall von Mais führt (Abb. 3, oben links), bringt der Ausschluss schlechter modellierter Spektralkanäle eine deutliche Verbesserung. In Abb. 3 oben Mitte wird nur der Chlorophyllsensitive Spektralbereich von 400 - $700 \mathrm{~nm}$ verwendet, der in dem Fall zufälligerweise fast dem optimalen Modell-Sampling entspricht (oben rechts): die am schlechtesten simulierten Spektralkanäle befinden sich im Red Edge Bereich (705 nm, 712 nm, 719 nm, 726 nm, 733 nm, 740 nm und 748 nm). Die vorgeschlagene Methodik verbesserte deutlich die Schätzung von LCC von einem rRMSE von 0.26 auf rRMSE von 0.15.

Bei Sojabohnen kommt es ebenfalls zu einer starken Überschätzung, wenn alle verfügbaren 73 Spektralkanäle verwendet werden (Abb. 3, unten links). Bei der Verwendung des sensitiven LCC Bereichs wird die Schätzung bereits verbessert von $\mathrm{rRMSE}=0.77$ auf $\mathrm{rRMSE}=0.41(\mathrm{Abb} .3$ unten Mitte). Deutlich weiter reduziert sich der Fehler dann durch die vorgeschlagene Optimierung der Modellkanäle, Abb. 3 unten rechts. Folgende Spektralkanäle wurden dabei eliminiert: $498 \mathrm{~nm}, 503 \mathrm{~nm}, 508 \mathrm{~nm}, 513 \mathrm{~nm}, 518 \mathrm{~nm}$,
$523 \mathrm{~nm}, 528 \mathrm{~nm}, 533 \mathrm{~nm}, 538 \mathrm{~nm}, 543 \mathrm{~nm}, 548 \mathrm{~nm}, 553 \mathrm{~nm}$, 698 nm, 705 nm 712 nm, 719 nm, 726 nm, 733 nm, 740 nm und $748 \mathrm{~nm}$. Zusammenfassend zeigen die Ergebnisse der LCC Schätzung eindeutig eine positive Auswirkung der Feature Selektion. Insbesondere durch die Entfernung von Spektralbändern mit relativ hohen Abweichungen zwischen Simulation und Messung, vor allem wenn der MAE $>0.01$ beträgt, können deutliche Verbesserungen erzielt werden. Die entfernten Kanäle befinden sich für beide Kulturen teilweise im gleichen Spektralbereich (Red Edge von $700-750$ nm), wo Chlorophyll eine stark abnehmende Absorption aufweist.

Man muss aber beachten, dass die LCC Schätzung nicht durch die alleinige Verwendung des sensiblen Spektralbereichs verbessert wird, sondern durch das Weglassen schlechter modellierter Banden. Solche schlecht modellierte Spektralkanäle beeinflussen das Ergebnis negativ. In einer Studie (Verrelst et al. 2016) wurde festgestellt, dass der Red Edge Bereich für die LCC-Schätzung durchaus wichtig ist, auch wenn es dort nur noch eine sehr schwache Absorption durch LCC gibt. Die Autoren erklärten dies durch Co-Varianzen mit anderen (strukturellen) Variablen, die im
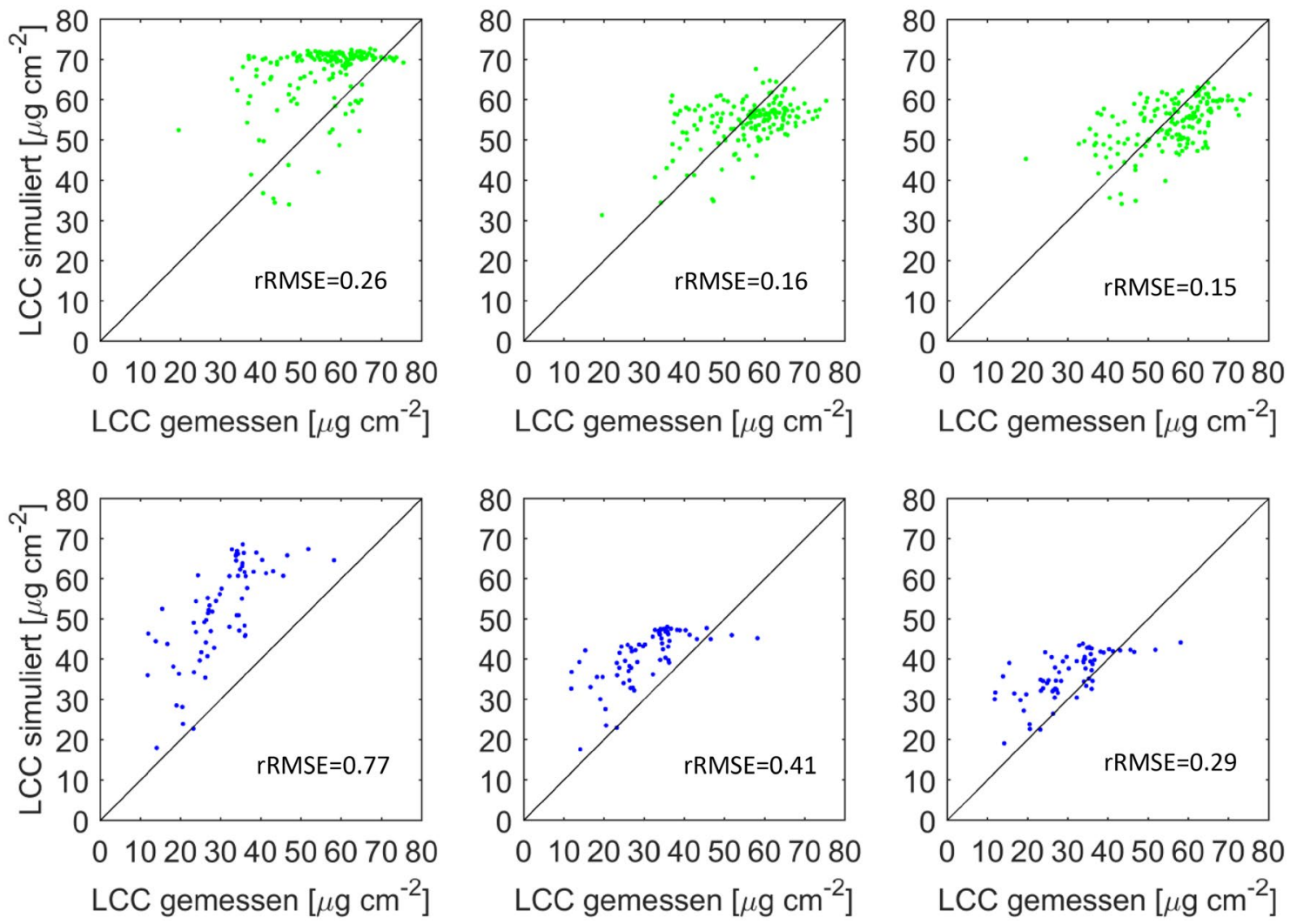

Abb. 3 Schätzgenauigkeit des Blattchlorophyllgehalts für Mais (obere Reihe; $\mathrm{N}=169$, grün) und Sojabohnen (untere Reihe; $\mathrm{N}=68$, blau) sowie verschiedenen spektralen Einstellungen. Links: unter Verwendung aller verfügbaren Banden $\mathrm{N}=73$. Mitte: nur Ver- wendung des sensitiven Spektralbereichs für LCC (400-700 nm, $\mathrm{N}_{\text {bands }}=51$ ). Rechts: unter Verwendung des optimalen spektralen Samplings. Fehlerstatistik: relativer RMSE (rRMSE) 
Red Edge sehr sensitiv sind (LAI). Die Studie basierte zwar ebenfalls auf dem Nebraska Datensatz. Es wurde aber ein statistisches FS-Verfahren verwendet, das sich alleinig an den gemessenen Variablen und nicht an den Spektren orientierte. In unserer Studie wird die Feature Selektion jedoch alleine durch das Modell gesteuert. Das bedeutet, dass die spektralen Samplings rein auf dem Vergleich von gemessenen und simulierten Reflektanzen beruhen. Ein hoher MAE führt zum Ausschluss eines Spektralkanals, unabhängig davon, ob er zur Variablenschätzung beiträgt. Dies zeigt auch das Beispiel von Sojabohnen: hier wurden sogar im blauen sichtbaren Spektralbereich, der wichtig für die LCC Schätzung ist, einige Banden eliminiert und somit die Schätzgenauigkeit erhöht (siehe Abb. 3 unten rechts).

Die unterschiedlichen Ergebnisse unserer Studie im Vergleich zur Studie von Verrelst et al. (2016) implizieren auch, dass die Ergebnisse immer vom verwendeten Algorithmus abhängen. Statistische Verfahren können mit einer geringeren Anzahl von Spektralkanälen dieselben oder sogar bessere Schätzergebnisse erzielen. Sie sind jedoch immer auf die Verfügbarkeit von gemessenen in situ Variablen angewiesen. Dieses Manko kann mit einem physikalisch-basierten oder hybriden Ansatz überwunden werden. Auffallend ist, dass die Streuung um die 1:1Linie hoch bleibt, selbst nach erfolgter Feature Selektion. Die erzielten optimalen rRMSE von 0.15 (Mais) und 0.29 (Sojabohnen) sind jedoch durchaus mit anderen Studien vergleichbar, zum Beispiel mit einem rRMSE von 0.19 in der vorher besprochenen statistischen Studie (Verrelst et al. 2016). Ungenauigkeiten in dieser Größenordnung können Einfluss auf die operative Verarbeitung und Weiterverwendung der Produkte nehmen.

Innovative multispektrale Erdbeobachtungsysteme wie die Sentinel-2 Sensoren der ESA ermöglichen bereits die akkurate Ableitung von Vegetationsprodukten. Hyperspektralsensoren mit einem quasi kontinuierlichen Spektralbereich werden die Erfassung zusätzlicher Vegetationseigenschaften ermöglichen. Dies kann insbesondere die Schätzung verschiedener biochemischer Komponenten betreffen, wie z.B. Chlorophyll-, Anthocyan-, Karotinoid- oder Proteingehalt, da sich deren Absorptionseigenschaften teilweise überlagern. Damit sind solche Blattinhaltsstoffe schwierig bzw. unmöglich durch (breite) multispektrale Daten zu differenzieren. Für eine Diskussion dieses Themas siehe auch Hank et al. (2018). Dieser Vorteil von hyperspektralen Daten gegenüber multispektralen Daten wurde bereits von anderen Studien anhand hyperspektraler Felddaten für LCC aber auch für andere Variablenschätzungen gefunden (Clevers and Kooistra 2012; Lee et al. 2004; Thorp et al. 2015). Unabhängig vom verwendeten System sollten vollständige Informationen über die Schätzunsicherheiten des Vegetationsprodukts bereitgestellt werden (Kaminski et al. 2017).

\section{Schlussfolgerung}

Zukünftige hyperspektrale Erdbeobachtungssysteme werden große Datenmengen generieren. Dies erfordert automatisierte und effektive Techniken für eine schnelle und effiziente Datenverarbeitung, beispielsweise für das Monitoring von Vegetationseigenschaften. Physikalisch-basierte Strahlungstransfermodelle haben in diesem Zusammenhang ein großes Potential. Bevor ein Strahlungstransfermodell wie PROSAIL zur Inversion herangezogen wird, sollte jedoch die Modelleignung im direkten Modus überprüft werden (Atzberger et al. 2013; Widlowski et al. 2015). Solche Tests helfen die Fähigkeit des Modells zu bewerten, die gemessene bi-direktionale Reflektanz des Bestandes im Vergleich zu den gemessenen Spektralwerten zu reproduzieren. Um BiasEffekte in der Schätzung zu vermeiden, sollten insbesondere Spektralkanäle mit einem systematischen Fehler entfernt werden.

Bisher wurde keine Studie durchgeführt, die das weit verbreitete PROSAIL-Modell zur Simulation hyperspektraler Messungen von zukünftigen HS Sensoren, z.B. HyspIRI, DESIS, PRISMA oder EnMAP, kritisch heranzog. Unsere Studie zeigt, dass PROSAIL in bestimmten Wellenlängenbereichen nicht optimal mit der gemessenen Reflexion von Mais, Sojabohne und Winterweizen übereinstimmt. Dies betrifft insbesondere den grünen Bereich $(500-550 \mathrm{~nm})$, sowie den Red Edge $(700-750 \mathrm{~nm})$. Die Probleme konnten durch die Anwendung des vorgeschlagenen LUT-basierten FS-Algorithmus minimiert werden. Durch die vollautomatisierte Elimination der Spektralkanäle mit MAE $>0.1$ zwischen simuliertem und gemessenem Signal, konnte die Schätzung für den Blattchlorophyllgehalt signifikant verbessert werden, teilweise in Kombination mit anderen Modellmodifikationen.

Die Ergebnisse der vorliegenden Studie beziehen sich ausdrücklich nicht auf die Empfindlichkeit der Variablen, sondern rein auf die Fähigkeit des Modells, spektrale Signaturen korrekt zu simulieren. Mögliche Gründe für die Nichtübereinstimmung können auf intrinsische Modelleigenschaften zurückgeführt werden. Diese Limitierungen sind derzeit Gegenstand weiterer Untersuchungen. In Abhängigkeit des aktuellen Wachstumsstadiums und der spezifischen Bestandsarchitektur können die Simulationen die tatsächlichen Messungen mit unterschiedlicher Genauigkeit darstellen. In diesem Zusammenhang muss darauf hingewiesen werden, dass auch die gemessenen Feldspektren Fehler enthalten. Für die vorliegende Studie wurden die gemessenen Spektraldaten jedoch als fehlerfrei angenommen. Eine Einbeziehung von Messunsicherheiten in den Inversionsprozess kann zu verbesserten Ergebnissen führen, z.B. durch Verwendung der Bayes'sche Statistik (Laurent et al. 2013). In der gleichen 
Logik ist auch zu berücksichtigen, dass in der vorliegenden Arbeit Fehler der atmosphärischen Korrektur nicht betrachtet wurden, wie auch Unterschiede in der räumlichen Auflösung.

Da für jede Feldfrucht spezifische „optimale“ Bandkombinationen identifiziert wurden, wäre für operationelle Anwendungen eine Kartierung der Feldfrüchte erforderlich. Dies ist prinzipiell möglich (Vuolo et al. 2018). Alternativ müsste ein (Kompromiss) Schwellwert definiert werden. Wir schlagen die Anwendung eines kulturübergreifenden Modellfehlers von $\mathrm{MAE}=0.01 \mathrm{im}$ VNIR Bereich zwischen gemessenen und modellierten Spektren vor, bevor PROSAIL invertiert wird. Um unsere Ergebnisse zu bestätigen, empfehlen wir, die Studie auf andere Standorte, Feldfrüchte und für andere Sensoren auszudehnen. Zusammenfassend kann unsere vorgeschlagene Feature Selektion eine effiziente Maßnahme zur Verbesserung der Simulationen von Strahlungstransfermodellen darstellen. Es wird empfohlen, diese vor der Anwendung von Inversionsstrategien für biophysikalische und biochemische Variablenschätzungen aus zukünftigen hyperspektralen Sensormissionen zu verwenden.

Acknowledgements Open access funding provided by University of Natural Resources and Life Sciences Vienna (BOKU).

Open Access This article is distributed under the terms of the Creative Commons Attribution 4.0 International License (http://creativecommons .org/licenses/by/4.0/), which permits unrestricted use, distribution, and reproduction in any medium, provided you give appropriate credit to the original author(s) and the source, provide a link to the Creative Commons license, and indicate if changes were made.

\section{Literatur}

Atzberger C, Richter K (2012a) Kombination spektraler und räumlicher Information für die fernerkundliche Erfassung von Vegetationsparametern. Vermessung \& Geoinformation 3:316-323

Atzberger C, Richter K (2012b) Spatially constrained inversion of radiative transfer models for improved LAI mapping from future Sentinel-2 imagery. Remote Sens Environ 120:208-218. https://doi. org/10.1016/j.rse.2011.10.035

Atzberger C, Darvishzadeh R, Schlerf M, Le Maire G (2013) Suitability and adaptation of PROSAIL radiative transfer model for hyperspectral grassland studies. Remote Sens Lett 4:56-65. https ://doi.org/10.1080/2150704x.2012.689115

Baret F, Andrieu B, Guyot G (1988) A simple model for leaf optical properties in visible and near-infrared: application to the analysis of spectral shifts determinism. In: Lichtenthaler HK (ed) Applications of chlorophyll fluorescence in photosynthesis research, stress physiology, hydrobiology and remote sensing. Springer, Dordrecht

Baret F, Buis S (2008) Estimating canopy characteristics from remote sensing observations: review of methods and associated problems. In: Liang S (ed) Advances in land remote sensing. Springer, Dordrecht. https://doi.org/10.1007/978-1-4020-6450-0_7. ISBN: 978-1-4020-6450-0

Berger K, Atzberger C, Danner M, D’Urso G, Mauser W, Vuolo F, Hank $\mathrm{T}$ (2018) Evaluation of the PROSAIL model capabilities for future hyperspectral model environments: a review study. Remote Sens 10:85

Botha EJ, Leblon B, Zebarth BJ, Watmough J (2010) Non-destructive estimation of wheat leaf chlorophyll content from hyperspectral measurements through analytical model inversion. Int J Remote Sens 31:1679-1697. https://doi.org/10.1080/01431160902926574

Ciganda V, Gitelson A, Schepers J (2008) Vertical profile and temporal variation of chlorophyll in maize canopy: quantitative "crop vigor" indicator by means of reflectance-based techniques. Agron J 100:1409-1417. https://doi.org/10.2134/agronj2007.0322

Clevers J, Kooistra L (2012) Using hyperspectral remote sensing data for retrieving canopy chlorophyll and nitrogen content. IEEE J Sel Top Appl Earth Obs Remote Sens 5:574-583. https://doi.org/10.1109/ jstars.2011.2176468

Darvishzadeh R, Skidmore A, Schlerf M, Atzberger C (2008) Inversion of a radiative transfer model for estimating vegetation LAI and chlorophyll in a heterogeneous grassland. Remote Sens Environ 112:2592-2604. https://doi.org/10.1016/j.rse.2007.12.003

Doktor D, Lausch A, Spengler D, Thurner M (2014) Extraction of plant physiological status from hyperspectral signatures using machine learning methods. Remote Sens 6:12247-12274. https://doi. org/10.3390/rs61212247

Dorigo W, Richter R, Baret F, Bamler R, Wagner W (2009) Enhanced automated canopy characterization from hyperspectral data by a novel two step radiative transfer model inversion approach. Remote Sens 1:1139-1170. https://doi.org/10.3390/rs1041139

D'Urso G et al (2010) Earth observation products for operational irrigation management in the context of the PLEIADeS project. Agric Water Manag 98:271-282. https://doi.org/10.1016/j.agwat .2010 .08 .020

Eckardt A, Horack J, Lehmann F, Krutz D, Drescher J, Whorton M, Soutullo M (2015) DESIS (DLR Earth Sensing Imaging Spectrometer for the ISS-MUSES platform). In: 2015 IEEE international geoscience and remote sensing symposium (IGARSS), 26-31 July 2015, pp 1457-1459. https://doi.org/10.1109/igars s.2015.7326053

España MAL, Baret F, Aries F, Chelle M, Andrieu B, Prévot L (1999) Modeling maize canopy 3D architecture: application to reflectance simulation. Ecol Model 122:25-43. https://doi.org/10.1016/S0304 -3800(99)00070-8

Féret JB, Gitelson AA, Noble SD, Jacquemoud S (2017) PROSPECT-D: towards modeling leaf optical properties through a complete lifecycle. Remote Sens Environ 193:204-215. https://doi. org/10.1016/j.rse.2017.03.004

Gitelson AA, Gritz Y, Merzlyak MN (2003) Relationships between leaf chlorophyll content and spectral reflectance and algorithms for nondestructive chlorophyll assessment in higher plant leaves. J Plant Physiol 160:271-282. https://doi.org/10.1078/0176-1617-00887

Godfray HCJ et al (2010) Food security: the challenge of feeding 9 billion people. Science 327:812-818. https://doi.org/10.1126/scien ce. 1185383

Guanter L et al (2015) The EnMAP spaceborne imaging spectroscopy mission for earth observation. Remote Sens 7:8830

Hank TB, Berger K, Bach H, Clevers JGPW, Gitelson A, Zarco-Tejada P, Mauser W (2018) Spaceborne imaging spectroscopy for sustainable agriculture: contributions and challenges. Surv Geophys. https:// doi.org/10.1007/s10712-018-9492-0

Jacquemoud S et al (2009) PROSPECT + SAIL models: a review of use for vegetation characterization. Remote Sens Environ 113(Supplement 1):S56-S66. https://doi.org/10.1016/j. rse.2008.01.026

Kaminski T, Pinty B, Voßbeck M, Lopatka M, Gobron N, Robustelli M (2017) Consistent retrieval of land surface radiation products from EO, including traceable uncertainty estimates. Biogeosciences 14:2527-2541. https://doi.org/10.5194/bg-14-2527-2017 
Kimes DS, Knyazikhin Y, Privette JL, Abuelgasim AA, Gao F (2000) Inversion methods for physically-based models. Remote Sens Rev 18:381-439. https://doi.org/10.1080/02757250009532396

Koetz B, Baret F, Poilvé H, Hill J (2005) Use of coupled canopy structure dynamic and radiative transfer models to estimate biophysical canopy characteristics. Remote Sens Environ 95:115-124. https:// doi.org/10.1016/j.rse.2004.11.017

Labate D et al (2009) The PRISMA payload optomechanical design, a high performance instrument for a new hyperspectral mission. Acta Astron 65:1429-1436. https://doi.org/10.1016/j.actaa stro.2009.03.077

Laurent VCE, Verhoef W, Damm A, Schaepman ME, Clevers JGPW (2013) A Bayesian object-based approach for estimating vegetation biophysical and biochemical variables from APEX at-sensor radiance data. Remote Sens Environ 139:6-17. https://doi. org/10.1016/j.rse.2013.07.032

Lee K-S, Cohen WB, Kennedy RE, Maiersperger TK, Gower ST (2004) Hyperspectral versus multispectral data for estimating leaf area index in four different biomes. Remote Sens Environ 91:508-520. https://doi.org/10.1016/j.rse.2004.04.010

Lee CM, Cable ML, Hook SJ, Green RO, Ustin SL, Mandl DJ, Middleton EM (2015) An introduction to the NASA Hyperspectral InfraRed Imager (HyspIRI) mission and preparatory activities. Remote Sens Environ 167:6-19. https://doi.org/10.1016/j.rse.2015.06.012

Locherer M, Hank T, Danner M, Mauser W (2015) Retrieval of seasonal leaf area index from simulated EnMAP data through optimized LUT-based inversion of the PROSAIL model. Remote Sens 7:10321-10346. https://doi.org/10.3390/rs70810321

Meroni M, Colombo R, Panigada C (2004) Inversion of a radiative transfer model with hyperspectral observations for LAI mapping in poplar plantations. Remote Sens Environ 92:195-206. https:// doi.org/10.1016/j.rse.2004.06.005

Richter K, Atzberger C, Vuolo F, Weihs P, D’Urso G (2009) Experimental assessment of the Sentinel-2 band setting for RTMbased LAI retrieval of sugar beet and maize. Can J Remote Sens 35:230-247

Rivera-Caicedo JP, Verrelst J, Muñoz-Marí J, Camps-Valls G, Moreno J (2017) Hyperspectral dimensionality reduction for biophysical variable statistical retrieval. Isprs J Photogramm 132:88-101. https ://doi.org/10.1016/j.isprsjprs.2017.08.012

Thorp KR, Gore MA, Andrade-Sanchez P, Carmo-Silva AE, Welch SM, White JW, French AN (2015) Proximal hyperspectral sensing and data analysis approaches for field-based plant phenomics. Comput Electron Agric 118:225-236. https://doi.org/10.1016/j.compa g.2015.09.005
Verger A, Baret F, Camacho F (2011) Optimal modalities for radiative transfer-neural network estimation of canopy biophysical characteristics: evaluation over an agricultural area with CHRIS/ PROBA observations. Remote Sens Environ 115:415-426. https:// doi.org/10.1016/j.rse.2010.09.012

Verhoef W, Jia L, Xiao Q, Su Z (2007) Unified optical-thermal fourstream radiative transfer theory for homogeneous vegetation canopies. IEEE Trans Geosci Remote Sens 45:1808-1822. https:// doi.org/10.1109/TGRS.2007.895844

Verrelst J, Rivera JP, Gitelson A, Delegido J, Moreno J, Camps-Valls G (2016) Spectral band selection for vegetation properties retrieval using Gaussian processes regression. Int J Appl Earth Obs Geoinf 52:554-567. https://doi.org/10.1016/j.jag.2016.07.016

Verrelst J et al (2018) Quantifying vegetation biophysical variables from imaging spectroscopy data: a review on retrieval methods. Surv Geophys 1:1. https://doi.org/10.1007/s10712-018-9478-y

Viña A, Gitelson AA, Nguy-Robertson AL, Peng Y (2011) Comparison of different vegetation indices for the remote assessment of green leaf area index of crops. Remote Sens Environ 115:3468-3478. https://doi.org/10.1016/j.rse.2011.08.010

Vohland M, Jarmer T (2008) Estimating structural and biochemical parameters for grassland from spectroradiometer data by radiative transfer modelling (PROSPECT + SAIL). Int J Remote Sens 29(1):191-209. https://doi.org/10.1080/01431160701268947

Vohland M, Mader S, Dorigo W (2010) Applying different inversion techniques to retrieve stand variables of summer barley with PROSPECT + SAIL. Int J Appl Earth Obs Geoinf 12(2):71-80

Vuolo F, Neuwirth M, Immitzer M, Atzberger C, Ng WT (2018) How much does multi-temporal Sentinel-2 data improve crop type classification? Int J Appl Earth Obs Geoinf 72:122-130

Weiss M, Baret F, Myneni RB, Pragnere A, Knyazikhin Y (2000) Investigation of a model inversion technique to estimate canopy biophysical variables from spectral and directional reflectance data. Agronomie 20:3-22

Widlowski J-L, Mio C, Disney M, Adams J, Andredakis I, Atzberger C, Brennan J, Busetto L, Chelle M, Ceccherini G et al (2015) The fourth phase of the radiative transfer model intercomparison (RAMI) exercise: actual canopy scenarios and conformity testing. Remote Sens Environ 169:418-437. https://doi.org/10.1016/j. rse.2015.08.016

Wocher M, Berger K, Danner M, Mauser W, Hank T (2018) Physicallybased retrieval of canopy equivalent water thickness using hyperspectral data. Remote Sens 10:1924

\section{Affiliations}

\section{Katja Berger ${ }^{1} \cdot$ Clement Atzberger $^{2} \cdot$ Martin Danner $^{1} \cdot$ Matthias Wocher $^{1} \cdot$ Wolfram Mauser $^{1} \cdot$ Tobias Hank $^{1}$}

Clement Atzberger

clement.atzberger@boku.ac.at

1 Department für Geographie, Ludwig-Maximilians Universität München, Luisenstr. 37, 80333 Munich, Germany
Institut für Vermessung, Fernerkundung und Landinformation, Universität für Bodenkultur Wien (BOKU), Peter-Jordan-Straße 82, 1190 Vienna, Austria 